\title{
Congenital prepubic sinus: An aborted dorsal urethral duplication or a cloacal remnant?
}

\section{Zafar Nazir}

Aga Khan University, zafar.nazir@aku.edu

Muhammad Arif Mateen Khan

Aga Khan University, arif.mateen@aku.edu

Javaria Qamar

Aga Khan University, javaria.qamar@aku.edu

Follow this and additional works at: https://ecommons.aku.edu/pakistan_fhs_mc_surg_paediatr

Part of the Pediatrics Commons, and the Surgery Commons

\section{Recommended Citation}

Nazir, Z., Khan, M., Qamar, J. (2018). Congenital prepubic sinus: An aborted dorsal urethral duplication or a cloacal remnant?. Journal of Pediatric Surgery, 53(7), 1-4.

Available at: https://ecommons.aku.edu/pakistan_fhs_mc_surg_paediatr/36 


\title{
Congenital prepubic sinus-An aborted dorsal urethral duplication or a cloacal remnant?
}

\author{
Zafar Nazir *, Muhammad Arif Mateen Khan, Javaria Qamar \\ Section of Pediatric Surgery, Department of Surgery, The Aga Khan University Hospital(AKUH), Karachi 74800, Pakistan
}

\section{A R T I C L E I N F O}

\section{Article history:}

Received 10 May 2018

Received in revised form 12 June 2018

Accepted 21 July 2018

Available online $\mathrm{xxxx}$

\section{Key words:}

Congenital prepubic sinus

Cloacal membrane

Sinus

Duplication of urethra

Infants and children

Congenital

\begin{abstract}
A B S T R A C T
Background: Congenital Prepubic Sinus (CPS) is an uncommon urogenital anomaly characterized by a blind tract between the skin over the pubis to anterior of the urinary bladder, Urethra or umbilicus. We report four such cases to emphasize varied clinical presentation and embryological conundrum.

Methods: Following Ethical Review Committee (ERC) approval, medical records of pediatric patients ( $<16$ years) presenting with CPS (identified through operating room records and Hospital Information Management System (HIMS) between 1994 and 2018 were reviewed for demographics, clinical presentation, investigations including histopathology, management and outcome.

Results: Four cases of CPS, 3 females and 1 male, age range 9 months to 13 years were managed over 25 -years. Clinical presentation includes a discharging sinus and recurrent episodes of cellulitis and abscess formation in pubic area and labia majora. Urological investigations were mostly normal. Insertion of lacrimal probe or plastic sheath of intravenous cannula through the sinus opening was useful to determine the course of sinus and aid its excision. Histology of excised sinus highlights the possible embryological origin.

Conclusions: CPS is a rare anomaly with varied clinical presentation. It seems CPS is an aborted urethral duplication (Stephen Type 3) or a Cloacal remnant. Complete excision of the tract in the reported cases was curative.

Type of study: Case series.

Level of evidence: IV
\end{abstract}

(c) 2018 Elsevier Inc. All rights reserved.
Congenital Prepubic Sinus (CPS), is a rare urogenital anomaly characterized by a blind epithelial lined tract, which usually extends from skin over the pubis close to the root of penis or clitoris to anterior of the urinary bladder, urethra or umbilicus. Usually there is no communication to urinary tract. After the first report by Campbell et al., around 50 cases are reported in literature [1-5]. We report 4 such cases managed at a university hospital in south-east Asia over 25-years (1994-2018), to highlight varied clinical presentation and embryological enigma.

\section{Material \& methods}

Following Institutional Ethical Review Committee (ERC) approval, medical records of pediatric patients $(<16$ years) presenting with CPS, between 1994 and 2018, were reviewed for demographics, clinical

\footnotetext{
* Corresponding author at: Section of Pediatric Surgery, Department of Surgery, The Aga Khan University, POB 3500, Stadium Road, Karachi 74800, Pakistan. Tel.: + 92213486 2358, + 923002128878 (Cell)

E-mail address: zafar.nazir@aku.edu (Z. Nazir).
}

presentation, investigations including histopathology, management and outcome.

\section{Case 1}

A 9-month old healthy girl presented with a discharging sinus at pubic area. The parents had observed occasional watery discharge from sinus opening since birth. Prior to presentation, she had two episodes of cellulitis adjacent to sinus opening and received antibiotics. There were no urinary symptoms. On examination there was a tiny opening in midline at pubis (Fig. 1a). Rest of the examination including external genitalia was unremarkable. Ultrasound of abdomen and pelvis showed normal urinary tract and Mullerian structures. Under general anesthesia a 24-size intravenous cannula sheath could easily be passed in the sinus tract, but contrast could not be injected. Cystoscopy revealed normal urethra and bladder. No internal opening or discharge of irrigation fluid was observed at the sinus opening. On exploration, a $4 \mathrm{~cm}$ tract was traced to bladder neck and excised (Fig. 1b). Histopathology showed the proximal lumen of the tract lined by transitional epithelium and distal lumen by stratified squamous epithelium surrounded by smooth muscle fibers with evidence of chronic inflammation and 

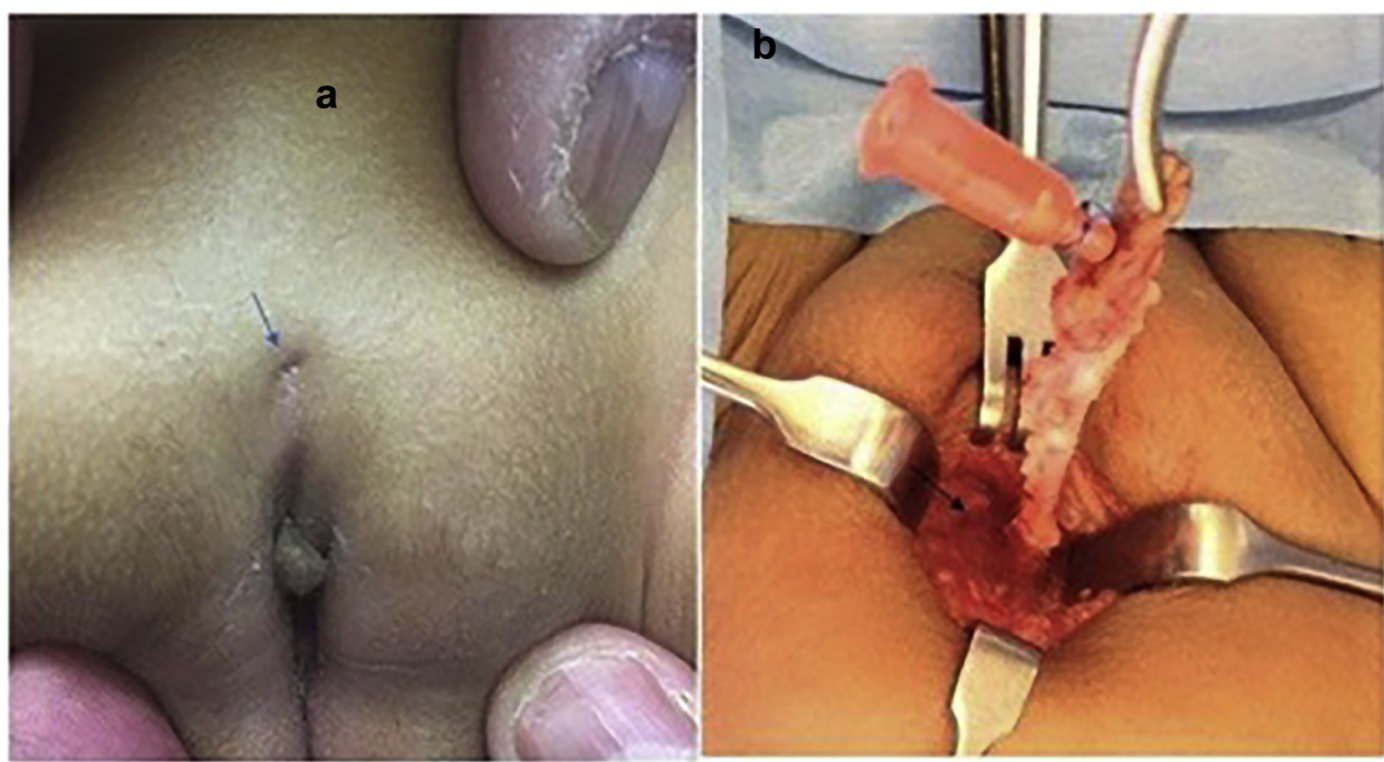

Fig. 1. a) Sinus opening at the root of clitoris (blue arrow), b) Intraoperative picture showing the sinus tract extending below the pubic symphysis (black arrow).

fibrosis (Fig. 2 a-c). She had an uneventful recovery and no recurrence observed at 9-month follow up.

\section{Case 2}

A 2-year old healthy boy presented with purulent discharge from an opening at the root of penis. He had incision and drainage of abscess at pubis twice in the preceding year and received broad-spectrum antibiotics for cellulitis around the sinus opening. There were no urinary symptoms and clinical examination was normal except a tiny opening at pubis. Ultrasound of abdomen, MCUG and Cystoscopy revealed normal bladder and urethra. A $4.5 \mathrm{~cm}$ fibrous tract passing below the pubic symphysis and terminating anterior to bladder neck was excised
(Table 1). Histological examination of excised tract showed lumen lined with admixture of stratified squamous, columnar and transitional epithelium surrounded by smooth muscle fibers and inflamed fibrous tissue. Postoperative recovery was uneventful and there was no evidence of recurrence on 3-year follow up.

\section{Case 3}

A 13-year old healthy girl presented with left labial abscess. She had incision and drainage of abscess and pus cultures revealed growth of Staphylococcus aureus. Two month later she presented with purulent discharge from $2 \mathrm{~mm}$ opening on the inner aspect of left labia and prominence of clitoris. She denied urinary symptoms. Urine culture

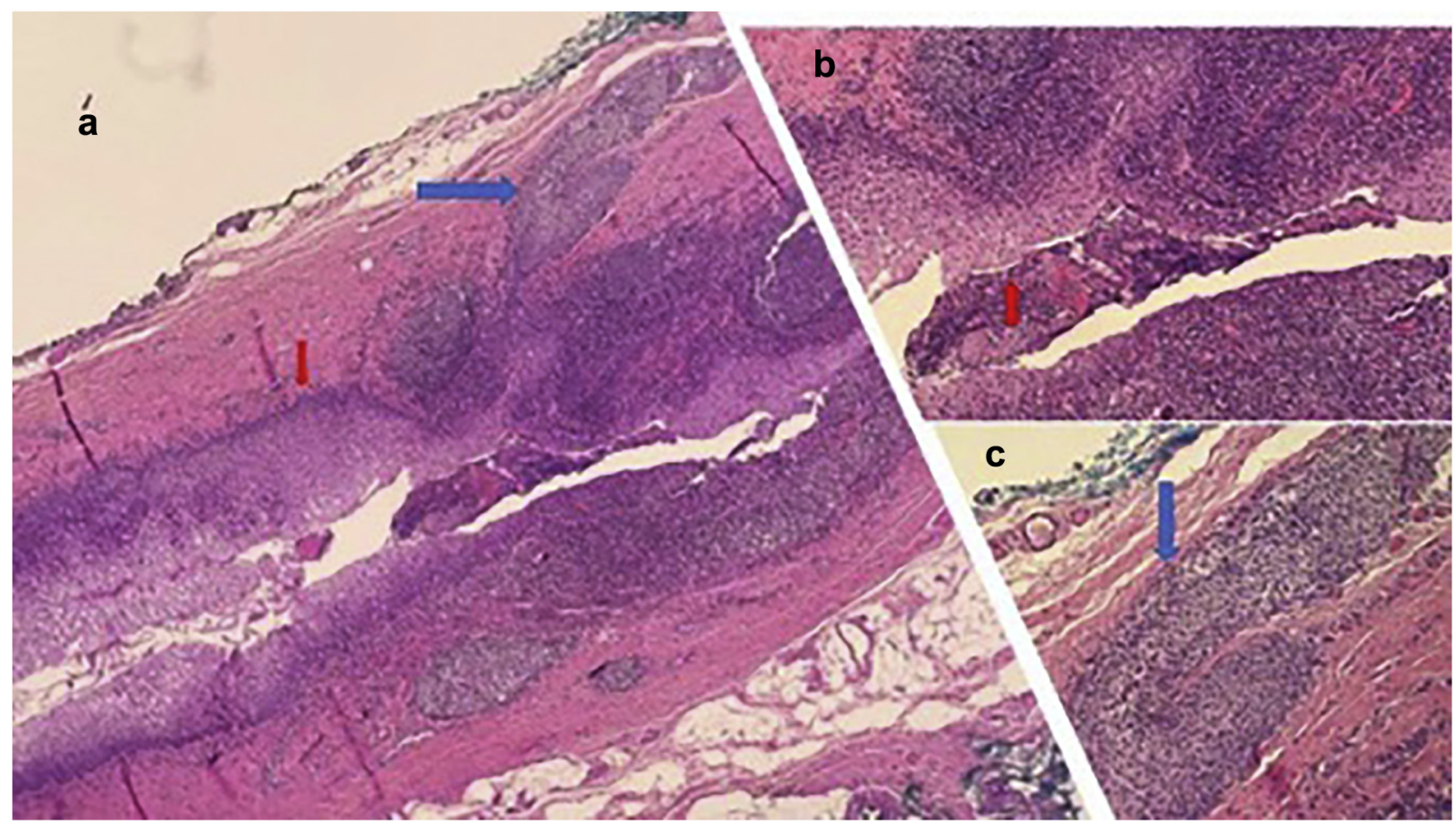

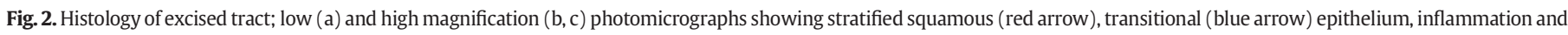
fibrosis. 
Table 1

Illustration showing the anatomical course (Red Line) of Congenital Prepubic Sinus in reported cases - Pubic bone (P), Urinary Bladder (UB), Uterus (U).

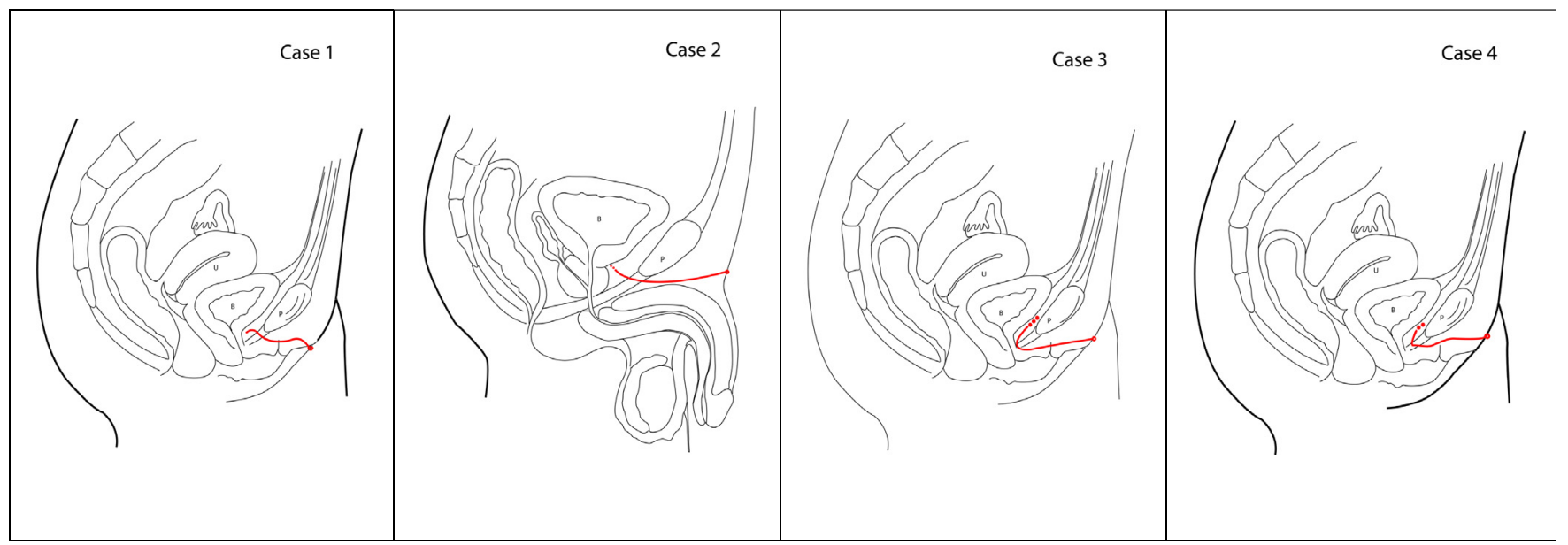

and Ultrasound of pelvis were unremarkable. A Sinogram revealed a blind tract ending in retropubic area with no communication to urinary tract. The sinus tract was excised. Histology revealed admixture of stratified squamous, columnar and transitional epithelium in the lining of tract (Table 1). Postoperative recovery was uneventful, and recurrence was not observed at 5-year follow up.

\section{Case 4}

An 18-month healthy girl presented with watery discharge from a tiny opening at pubis. Clinical Examination, Ultrasound abdomen and MCUG were normal. At surgery, a sinus tract was dissected down to the bladder, transfixed and excised. There was no communication to urinary tract (Table 1). On histology the tract was lined by admixture of transitional, columnar and squamous epithelium and surrounded by smooth muscle scattered in fibrous tissue. The patient recovery was uneventful and on 2-year follow-up there was no evidence of recurrence.

\section{Discussion}

Congenital Prepubic Sinus (CPS), is a blind tract extending from skin overlying pubis adjacent to the base of penis or clitoris to (but not communicating with) urinary bladder, bladder neck or umbilicus. It is also reported in literature as suprapubic fistula, suprapubic dermoid sinus etc. After the first report by Campbell et al., in 1987, around 50 cases are reported in literature [1-5]. Four theories are proposed to explain the etiology of CPS: 1) anomalous abdominal wall closure; 2 ) a variant of dorsal urethral duplication; 3 ) a fistula of primitive urogenital sinus and 4) a remnant of primitive cloaca [6-9]. Routine and immunohistochemical staining of tract in patients reported here and in literature; specifically, the reports by Huang et al., Chou et al. and Balster et al., validates the presence of transitional, columnar and squamous epithelium in the lining. It seems that CPS is an aborted dorsal urethral duplication as described by Stephen et al., (type 3) or a cloacal remnant [10-16]. Stratified squamous and transitional epithelium can be found in urinary system (allantois) but columnar epithelium originates only from hindgut. Thus, the most likely source of 3 different type of epithelium in the lining of sinus is residual cloacal membrane [11]. It is postulated by Tsukamoto et al. that during the embryonic development, the umbilicophallic groove traps a small share of cloacal membrane during its migration from the base of umbilical cord and tubularization following longitudinal growth of embryo [8]. The degree of depth of trapped cloacal membrane can explain the variable direction and location of blind ending tract at umbilicus, bladder or urethra. Residual membrane above the genital tubercle may interrupt the complete fusion of pubic symphysis as pubic diastasis is seen in the reported cases $[1,17]$.

CPS usually presents in infancy and young children (preponderance in females) with persistent discharging sinus in pubic region close to the root of penis or clitoris. Three out of four patients in this series were females. Superimposed bacterial infection is common and is difficult to eradicate with broad-spectrum antibiotics and minor surgical procedures as two of our patients persist to have sinus after incision and drainage of abscess. Late presentation with labial abscess and Clitoromegaly in adolescence is unusual $[18,19]$. Ultrasound examination of urinary tract, Voiding Cystourethrogram (VCUG) and Cystoscopy are usually normal. Conventional Fistulography and fine lacrimal probe or plastic sheath of intravenous cannula are useful to delineate the anatomy [20]. However, it is arduous to perform contrast study as sinus tract is usually blind and narrow, and is not in communication with urinary tract. Sasaki et al., and Yamada et al., have reported the superiority of MRI in delineating the anatomy of tract and it may be the diagnostic study of choice in future $[21,22]$.

Complete and meticulous excision of tract (owing to its variable direction and location) is required to prevent recurring symptoms, infections and potential for late malignant changes [20]. A fine lacrimal probe or an intravenous cannula as illustrated in Fig. 1b, can facilitate complete excision of the tract.

In conclusion, CPS is a rare genitourinary anomaly of pediatric age. It is most likely a remnant of cloacal membrane as is supported by literature and our experience of four cases. Careful and complete excision is usually curative.

\section{References}

[1] Campbell J, Beasley S, Mcmullin N. Congenital prepubic sinus: possible variant of urethral duplication (Stephen type 2). J Urol 1987;137:505-6.

[2] Özdemir E, Yldz T, Kanbay M, et al. A case of congenital prepubic sinus. Eur J Pediatr Surg $2011 ; 21: 408-9$.

[3] Sakaguchi T, Hamada Y, Nakamura Y, et al. Congenital prepubic sinus: a case report and review of the literature. J Pediatr Surg Case Rep 2016;8:22-6.

[4] Aihole JS, Narendra Babu M, Javaregowda D, et al. Congenital prepubic sinus-a variant of urethral duplication. Afr J Urol 2016;22:196-8.

[5] Nonnenmacher G, Rhein D, Loff S. Congenital prepubic sinus: rare diagnosis with unclear etiology. J Pediatr Surg Case Rep 2016;9:16-8.

[6] Sinha AK, Kumar B, Kumar P, et al. Congenital pre-pubic sinus: an embryological enigma. J Pediatr Surg Case Rep 2017;18:36-8.

[7] Soares-Oliveira M, Juliá V, Aparicio LG, et al. Congenital prepubic sinus. J Pediatr Surg 2002;37:1225-7.

[8] Tsukamoto K, Yamataka A, Kuga T, et al. Congenital prepubic sinus: is it a residual cloacal membrane and umbilicophallic groove? Pediatr Surg Int 2004;20:47-50. 
[9] Stephen FD. Abnormal embryology-cloacal dysgenesis. Congenital malformations of urinary tract. New York: Praeger Scientific; 1983. p. 22.

[10] Huang CC, Wu WH, Chai CY, et al. Congenital prepubic sinus: a variant of dorsal urethral duplication suggested by immunohistochemical analysis. J Urol 2001;166:1876-9.

[11] Chou TD, Chu CC, Diau GY, et al. Subpubic sinus: a remnant of cloaca. J Urol 1995; 153:1671-2.

[12] Balster S, Bettendorf O, Brinkmann OA, et al. Congenital prepubic sinus: etiology and therapy. Aktuelle Urol 2003;34:484-7.

[13] Komura J, Yano H, Kanazawa M, et al. Congenital prepubic sinus. Pediatr Surg Int 1994;9:287-9.

[14] Al-Wattar KMS. Congenital prepubic sinus: an epispadiac variant of urethral duplication: case report and review of literature. J Pediatr Surg 2003;38:E10. https://doi. org/10.1053/jpsu.2003.50148.

[15] Ergun O, Sayan A, Arikan A. Congenital prepubic sinus: possible variant of dorsal urethral duplication. Eur J Pediatr Surg 1998;8:380-1.
[16] Nasir AA, Abdul-Rehman LO, Olaoye I, et al. Congenital prepubic sinus: a varient of epispadias dorsal urethral duplication.J Pediatr Urol 2013:9:e82-5.

[17] Daher P, Diab N, Moussa C, et al. Congenital prepubic sinus. Eur J Pediatr Surg 1994; 4:119-21. https://doi.org/10.1055/s- 2008-1066083.

[18] Shaw JS, Calldmone AA, Rardin CR. Congenital prepubic sinus in an adolescent female: a case report and review of literature. Urology 2015;85:227-9.

[19] Hayase M, Kojima Y, Hayashi Y, et al. Pseudo-clitoromegaly associated with congenital prepubic sinus. Int J Urol 2006;13:1031-2.

[20] Park WH, Choi SO. An unusal urachal sinus with external opening in the midline suprapubic area. J Pediatr Surg 2003;38:E22. https://doi.org/10.1016/S0022-3468 (03)00216-1.

[21] Sasaki Y, Deguchi E, Tsukada S, et al. Usefulness of magnetic resonance imaging for congenital prepubic fistula. J Pediatr Surg 2010;45:1734-6.

[22] Yamada K, Kanamori Y, Tanaka H, et al. Congenital prepubic sinus associated with a urachal remnant: report of a case. Surg Today 2013;43:1330-2. 